\begin{tabular}{|c|c|}
\hline Version & Authors original manuscript after peer review \\
\hline Title & $\begin{array}{l}\text { Effect of blending on the chain dynamics of the "Low-Tg" } \\
\text { component in nonentangled and dynamically asymmetric polymer } \\
\text { blends }\end{array}$ \\
\hline Authors & $\begin{array}{l}\text { Arrese-Igor, Silvia; Alegria, Angel; Moreno Segurado, Ángel J.; } \\
\text { Colmenero, Juan }\end{array}$ \\
\hline & Publisher data \\
\hline Issue date & 2011 \\
\hline Publisher & American Chemical Society \\
\hline Gitation & Macromolecules 44(9): $3611-3621$ (2011) \\
\hline Publisher Version & http://dx.doi.org/10.1021/ma2000064 \\
\hline URI: & http://hdl.handle.net/10261/48252 \\
\hline DOI: & http://dx.doi.org/10.1021/ma2000064 \\
\hline ISSN & $0024-9297$ \\
\hline E-ISSN: & $1520-5835$ \\
\hline
\end{tabular}




\title{
Effect of blending on the chain dynamics of the "Low-Tg" component in non-entangled and dynamically asymmetric polymer blends
}

\author{
S. Arrese-Igor ${ }^{1}$, A. Alegría ${ }^{1,2}$, A. J. Moreno ${ }^{1}$ J. Colmenero ${ }^{1,2,3}$ \\ 1 Centro de Física de Materiales (CSIC-UPV/EHU), \\ Paseo Manuel de Lardizabal 5, 20018 San Sebastián, Spain \\ 2 Departamento de Física de Materiales UPV/EHU, \\ Paseo Manuel de Lardizabal 3, 20018 San Sebastián, Spain and \\ 3 Materials Physics Center (MPC), and Donostia International Physics Center, \\ Paseo Manuel de Lardizabal 4, 20018 San Sebastián, Spain
}

(Dated: March 18, 2011)

We have studied polyisoprene (PI) component chain dynamics in poly-tertbutylstyrene (PtBS) miscible blends of low molecular weight over the entire composition range by broadband dielectric spectroscopy (DS). Blends with two different molecular weight of PtBS, $\overline{M_{n}}=1300 \mathrm{Da}$ and $\overline{M_{n}}=2300 \mathrm{Da}$, having notably different glass transition temperatures, $T_{g}=330 \mathrm{~K}$ and $T_{g}=373 \mathrm{~K}$ respectively, have been investigated. The molecular weight for PI was $\overline{M_{n}}=2700 \mathrm{Da}$, and $T_{g}=204 \mathrm{~K}$, i.e. well below the $T_{g}$ of PtBS. The molecular weight of all the polymers were chosen in order to be well below the entanglement limit for PI. In addition to the slowing down of the dielectric response of PI as the high- $T_{g}$ component PtBS increases, there is a gradual broadening of both low and high frequency tails of the normal mode relaxation as PtBS\% increases, and for a given composition as the temperature decreases. 
The magnitude of the broadening depends on: i) the concentration; ii) the temperature; iii) the dynamic asymmetry, i.e. the difference between the pure component's $T_{g}$. The onset of the mentioned broadening correlates well with the freezing of the segmental relaxation of the high- $T_{g}$ PtBS component. Finally, the terminal dynamics of PI component shows a stronger T-dependence than its segmental dynamics and the effect is more pronounced the higher the PtBS content. As a result, the separation between the maxima of both relaxations ranges from 3 decades in pure $\mathrm{PI}$ and $\mathrm{PI} \geq 50 \%$ content blends, to $\sim 5$ decades for $20 \% \mathrm{PI}$ blends. The results are discussed in terms of the dynamic asymmetry between the different blend components and compared with simulation results of dynamically asymmetric bead-spring polymer blends showing anomalous scaling behavior of the Rouse modes. 


\section{INTRODUCTION}

Dynamics in polymer blends is a very important topic, not only from a fundamental point of view, but also for technological applications. The mixing of two compatible polymers is one of the most cost-effective ways to tune the properties of available polymeric materials to meet application needs. A limited knowledge of the blending phenomenon restricts the advance in the pursuit of taylor-made materials so a deep molecular level understanding is crucial. Miscible polymer blends have been known for some time to exhibit striking dynamic phenomena (see e.g. reviews ${ }^{1-3}$ and references therein). Concerning the segmental dynamics driving the so-called $\alpha$-relaxation, two main effects are well established by extensive experimental studies: i) the isolated dynamical response of each component broadens with respect to the pure polymer behavior, this effect being strongly dependent on temperature (it tends to disappear at high $\mathrm{T}$ ) and more evident for the component with the lower glass transition temperature, $T_{g}$; and ii) two distinct mean relaxation times are usually found in the blend, each of them corresponding to the dynamics of each component modified by blending, what is usually called 'dynamic heterogeneity'. Confinement effects in dynamically asymmetric blends is also a hot topic which arouses large amount of research. When the slower component of a miscible blend dynamically freezes, the segmental relaxation of the faster component has been reported to show a crossover to a more localized and faster dynamics ${ }^{4-11}$, in analogy with the behavior shown by diverse systems confined within well-defined geometries ${ }^{12-14}$.

In addition to the rationalization of component segmental dynamics in miscible blends, it is also of great importance to understand the effect of blending on the viscoelastic relaxation of each species and on the rheological properties of the blend. 'Dynamic heterogeneity' 
in blends extends to terminal relaxation and two distinct mean relaxation times have been found, each of them corresponding to the dynamics of each component modified by blending ${ }^{15-19}$. For a pure homopolymer well above $T_{g}$ the segmental and terminal relaxation times usually show similar T-dependence ${ }^{20,21}$. The Rouse model ${ }^{22}$, which is based on a bead-spring description of the polymer chain under random external forces, successfully reproduces this connection between the two dynamics. As a consequence, attempts have been made to predict and describe the rheological properties of the blend starting from the segmental relaxation of each component under the assumption that the terminal and segmental dynamics for a given component in a miscible blend have the same T-dependence ${ }^{23,24}$. The compilation of literature data, however, indicates that the terminal dynamics does not track the segmental dynamics in all miscible polymer blends ${ }^{15,16,19,25}$.

To date, most of the systems used to learn how component chain dynamics are modified by blending, had relatively high molecular weights. High molecular weight systems are closer to practical applications and more interesting from a rheological point of view, but complicate the analysis and identification of fundamental relations as even high molecular weight homopolymers depart from the Rouse behavior when topological constraints due to chain entanglements come into play. In this work we have studied the component dynamics of PI in PI/PtBS miscible blends of low molecular weight - well below the entangled limitby dielectric spectroscopy over the entire composition range. The suitability of the Rouse model to describe the chain dynamics of this low molecular weight PI has been carefully checked recently ${ }^{26}$. Results in the present paper show that time temperature superposition of the normal mode relaxation of PI in the blends does not hold on either the low or the high frequency tails of the dielectric relaxation spectra. Moreover, the comparison of normal mode and $\alpha$-relaxation times for PI indicates that they do not track each other, and become 
more and more apart the lower the PI content. The data presented herein evidence the non-trivial character of blending phenomena even for this relatively simple case. Finally, an attempt to describe the dielectric data in terms of non-exponential Rouse modes is made by comparison with the results of simulations of mixtures of short bead-spring polymer chains with high dynamic asymmetry.

\section{EXPERIMENTAL}

\section{A. Samples}

Samples were prepared starting from commercially available pure homopolymers Polyisoprene (PI) and Poly-tert-butylstyrene (PtBS) purchased from Polymer Source. The low- $T_{g}$ component of the blends was PI ( $80 \%$ cis 1,$4 ; 15 \%$ trans 1,$4 ; 5 \%$ trans 3,4$)$ with $\overline{M_{n}}=2700$ Da and $\overline{M_{w}} / \overline{M_{n}}=1.07$ (PI27 from now on) and a glass transition temperature, $T_{g}=204 \mathrm{~K}$ determined by differential scanning calorimetry. Two PtBS with different molecular weights and $T_{g}$ s were used as high- $T_{g}$ components: $\operatorname{PtBS} 13\left(\overline{M_{n}}=1300 \mathrm{Da} ; \overline{M_{w}} / \overline{M_{n}}=1.08\right)$ and PtBS23 $\left(\overline{M_{n}}=2300 \mathrm{Da} ; \overline{M_{w}} / \overline{M_{n}}=1.06\right)$ with $T_{g}=330 \mathrm{~K}$ and $T_{g}=373 \mathrm{~K}$ respectively. The $T_{g}$ difference between the PtBS samples is large since the molecular weights are relatively low ${ }^{26}$. The molecular weight for all the polymers were chosen in order to be well below the entanglement limit for PI, which is around 4000 Da. PI/PtBS blends show Low Critical Solution Temperature behavior, being miscible below $443 \mathrm{~K}^{17,27}$. Starting from the homopolymers, blends with several PI concentrations (weight content) were prepared by solution casting from toluene solutions. Prior to measurements samples were vacuum dried at temperatures above the glass transition to completely eliminate any trace of solvent. All the blends were transparent and showed no crystallization or melting by calorimetry. Following the 
same procedure additional blends with a high molecular weight PI, $\overline{M_{n}}=76500 \mathrm{Da}$ and $\overline{M_{w}} / \overline{M_{n}}=1.07$ (PI765 from now on) and high PtBS concentration (80\%) were prepared to study the dielectric $\alpha$-relaxation of the PtBS and PI components.

The calorimetric glass transitions of the blends were very broad, extending over more than 60K. This phenomenology has often been reported for polymer blends in general, and in particular, for blends with high dynamical heterogeneity as those studied here. By differential scanning calorimetry, the heat-flow temperature derivative evidences two overlapped peaks which can be identified with the effective glass transition of each component in the blend. The complete calorimetric characterization of the samples was the subject of a separate communication $^{28}$, where good correspondence between the effective $T_{g, e f f} \mathrm{~S}$ of the different components measured by calorimetric and dielectric spectroscopy techniques was found.

\section{B. Dielectric Spectroscopy}

A broadband dielectric spectrometer, Novocontrol Alpha-A analyser, was used to isothermaly measure the complex dielectric function $\epsilon^{*}(\omega)=\epsilon^{\prime}(\omega)-i \epsilon^{\prime \prime}(\omega), \omega=2 \pi f$, between 130 and $350 \mathrm{~K}$, and in the $10^{-2}$ to $10^{7} \mathrm{~Hz}$ frequency range, by placing samples between parallel gold-plated electrodes of $30 \mathrm{~mm}$ diameter. The temperature stability was better than $\pm 0.2 \mathrm{~K}$. As cis-PI is type-A polymer having components of the dipole moment parallel and perpendicular to the chain, both chain and segmental relaxations can be followed by dielectric spectroscopy. The dielectric losses of PI are almost two orders of magnitude larger than those of PtBS. As a result, when dielectricaly measuring PI/PtBS blends, we will be mainly sensitive to the PI component dynamics in the blend.

The measured spectra in general show two partially overlapped relaxation processes corresponding to the main chain (normal mode -NM-) and the segmental ( $\alpha$ ) relaxation of PI27 


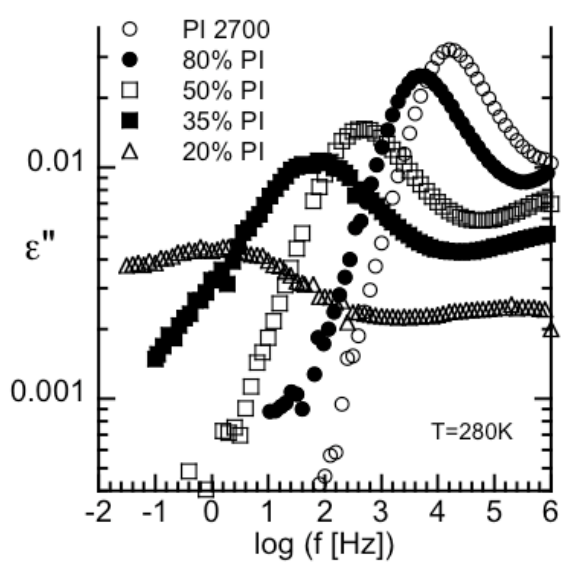

FIG. 1: Imaginary part of the relative dielectric permitivity of pure PI27 and its blends with PtBS13 at different weight percentages at 280K.

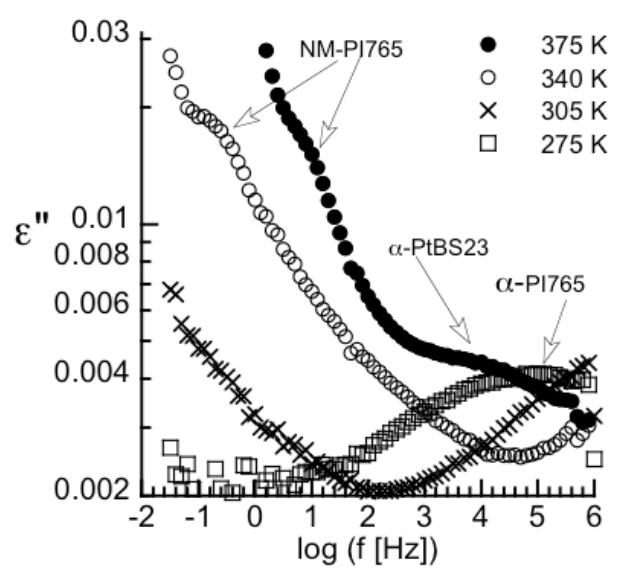

FIG. 2: Imaginary part of the relative dielectric permitivity of $20 \%$ PI765 blend with PtBS23 at different temperatures.

in the blends (see figure 1 for example) and no evidence of PtBS's loss peak can be detected. With decreasing PI content, the intensity and characteristic frequencies of these relaxations decrease. Regarding the shape of the relaxations, we found a gradual broadening of both processes with decreasing PI content, which makes the maxima of both relaxation to be 
less and less pronounced the lower the PI\%. As can be seen, this effect is dramatic for the $20 \%$ PI blends.

On the other hand, the $\alpha$ and NM-relaxations of samples prepared with the high molecular weight PI765 appear well separated (see figure 2). Due to the longer chain length of PI765, the NM-relaxation of PI moves to much lower frequencies revealing: (i) the low frequency part of the $\alpha$-relaxation of PI in the blend; and (ii) for samples with the highest PtBS content a weak contribution from the $\alpha$-relaxation of the PtBS component in the blend, otherwise masked by PI27's NM (see figure 2). In the following, we will mainly focus on the study of the NM process as the characterization of the $\alpha$-relaxation was the subject of a separate communication $^{28}$.

\section{Effect of blending on the NM-relaxation of PI}

As we have already mentioned, there is a clear slowing down of the NM-relaxation of PI in the blend together with a monotonic decrease of its intensity as PtBS -high- $T_{g}$ componentcontent increases. Figure 1 shows the imaginary part of the relative dielectric permitivity of pure PI27 and its blends with PtBS13 at different weight percentages at $280 \mathrm{~K}$. The results for PI27/PtBS23 blends look pretty much the same. The temperature dependence of the characteristic frequency of the NM-relaxation obtained from the position of the dielectric loss maxima are shown in figure 3 for PI27 and its blends with PtBS13 and PtBS23, panels (a) and (b) respectively. Curves in figure 3 were fitted to a Vogel-Fulcher-Tamman law (VFT),

$$
\log f_{\text {max }}=\log f_{\infty}-\frac{B}{2.3 *\left(T-T_{o}\right)},
$$


where $\log f_{\infty}$ was fixed to the value obtained for the pure homopolymer. The resulting parameters have been included in figure 4 , where a change in the trend of both $T_{o}$ and $B / T_{o}$ can be observed for $\mathrm{PI} \%<50$.

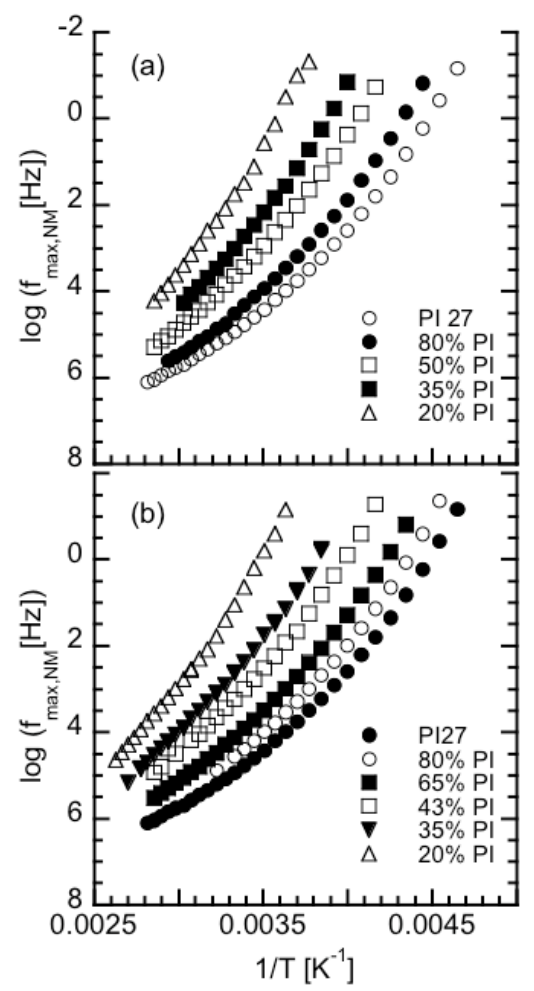

FIG. 3: Logarithm of the frequency at the maxima of the normal mode relaxation of pure PI27 and its blends with PtBS13 (a) and PtBS23 (b) at different PI weight percentages.

In addition to the slowing down of the NM, it is clear from figure 1 that the NM-relaxation of PI in the blends is broader than that of the pure homopolymer, being wider the higher the PtBS content. Moreover, the width of the NM is temperature dependent, so that a similar gradual broadening can be observed for a fixed composition when decreasing temperature. As a consequence, the time temperature superposition (tTS) of the normal mode relaxation of PI in the blends does not hold. Figure 5a shows in a logarithmic scale the 


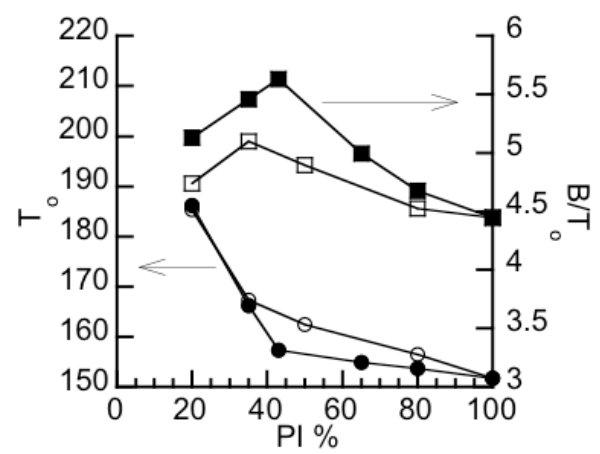

FIG. 4: Parameters for the VFT fitting of the NM-relaxation of PI in its blends with PtBS13 (empty symbols) and PtBS23 (filled symbols) at various concentrations.

imaginary part of the relative dielectric permitivity at different temperatures for the sample 35\% PI27/PtBS23. Figure 5b shows in a linear scale the imaginary part of the relative dielectric permitivity at different temperatures normalized to maxima position and intensity of the NM for the sample 43\%PI27/PtBS23. The decrease of the steepness of the loss spectra on its low frequency side as $\mathrm{T}$ decreases is clear in both samples. Analogous broadening at the high frequency side of the NM-relaxation is also evident in the $43 \% \mathrm{PI} 27 / \mathrm{PtBS} 23$ sample, see figure 5b. This high frequency broadening is also present in the lower PI content samples, but the closer and likewise broadened $\alpha$-relaxation in these samples prevents from seeing this same effect in such a clear way. In order to further check whether similar broadening of the high frequency tail of the NM exists in these low PI27 content blends, we have chosen to experimentally characterize the $\alpha$-relaxation of PI765/PtBS blends instead of a 'fitting' procedure of the overlapped signals of PI27/PtBS blends. In this way the analysis will be less model dependent. For that we have measured the $\alpha$-relaxation in blends with PI765, where the NM and the $\alpha$-relaxation stay far separated. The $T_{g}$ temperatures of the low and high molecular weight pure PI are slightly different ${ }^{26}$, however, blending makes 

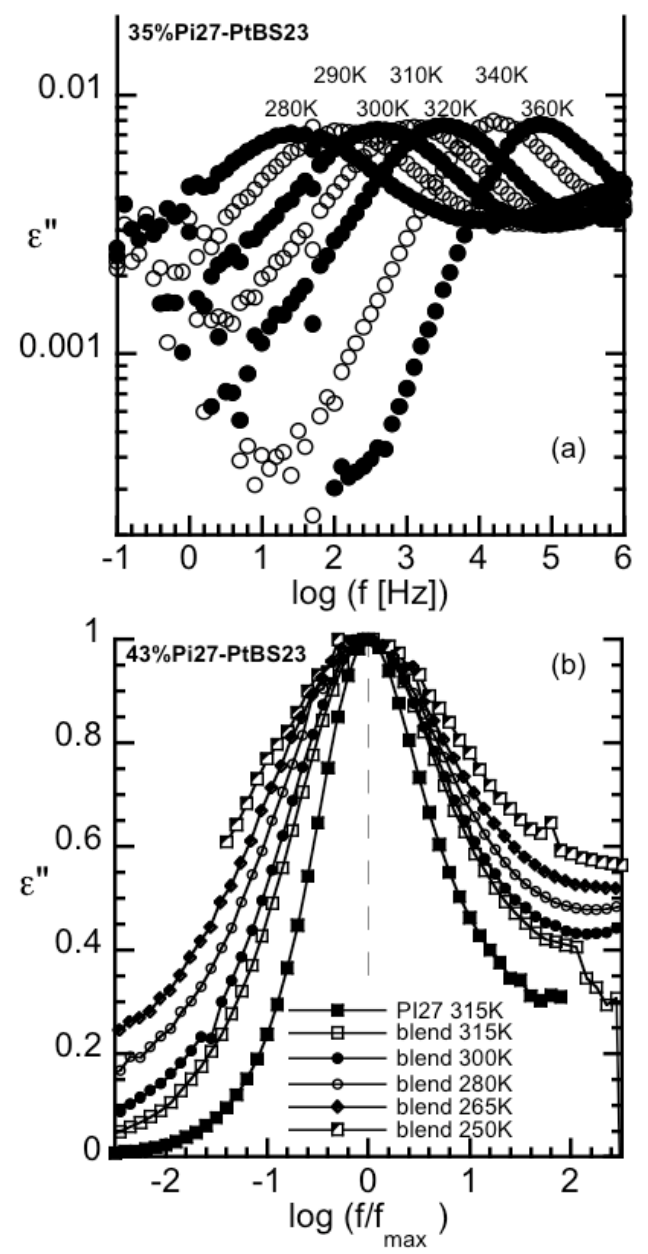

FIG. 5: Imaginary part of the relative dielectric permitivity of: (a) 35\%PI27/PtBS23 blend at temperatures between $360 \mathrm{~K}$ and $280 \mathrm{~K}$; and (b) 43\%PI27/PtBS23 blend normalised to maxima position and intensity.

the difference between the effective glass transitions of PI27 and PI765 in the blends even smaller. As a result, and due to the broad character of the $\alpha$-relaxation both PI27 and PI765 seem to show indistinguishable relaxations within the experimental error. Figure 6 shows for two selected temperatures the dielectric relaxation of the 20\%PI27/PtBS23 (empty circles) and 20\%PI765/PtBS23 (empty squares) blends, together with the result of subtracting to 
the $20 \%$ PI27/PtBS23 blend the $\alpha$-relaxation of PI obtained from 20\%PI765/PtBS23 sample measurements (filled circles). The whole set of NM-relaxation curves obtained by this procedure for the 20\%PI27/PtBS23 blend are depicted in figure 7 . From these results, the broadening of the NM at the high frequency side is evident also for low PI\% samples. It is worth mentioning that the broadening effect is higher for PtBS23 blends than for PtBS13 ones, i.e. it increases with the difference between homopolymer glass transition temperatures and consequently with the dynamic asymmetry. Thus results in the present paper show that time temperature superposition of the normal mode relaxation of PI in the blends does not hold on either the low or the high frequency tails of the relaxation.

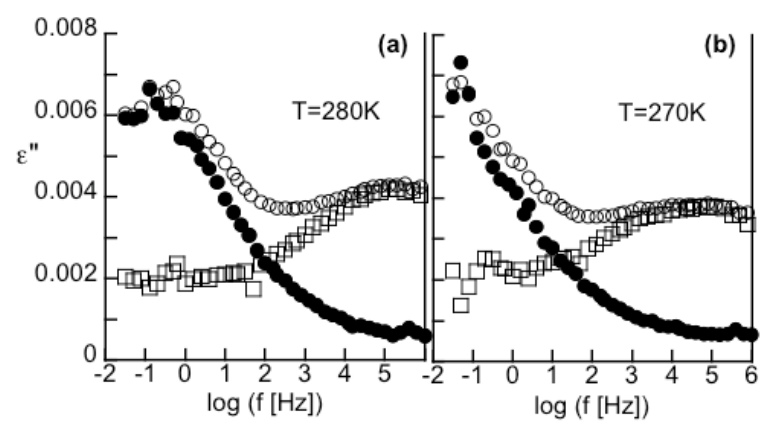

FIG. 6: Imaginary part of the relative dielectric permitivity for 20\%PI/PtBS23 blends with two different PIs: low molecular weight PI27 (empty circles) and high molecular weight PI765 (empty squares) at 270 and $280 \mathrm{~K}$. Filled circles represent the dielectric response of the low molecular weight 20\%PI27/PtBS23 blend after subtracting the $\alpha$-relaxation from the high molecular weight sample.

The broadening, although more evident the lower the PI content, is also present for blends with medium PI concentrations and it is greatly dependent on temperature, as it can be seen in figure 8 for the two series of blends studied. For the characterization of this broadening we determined the half width at half maxima (HWHM) from the low frequency side of the 


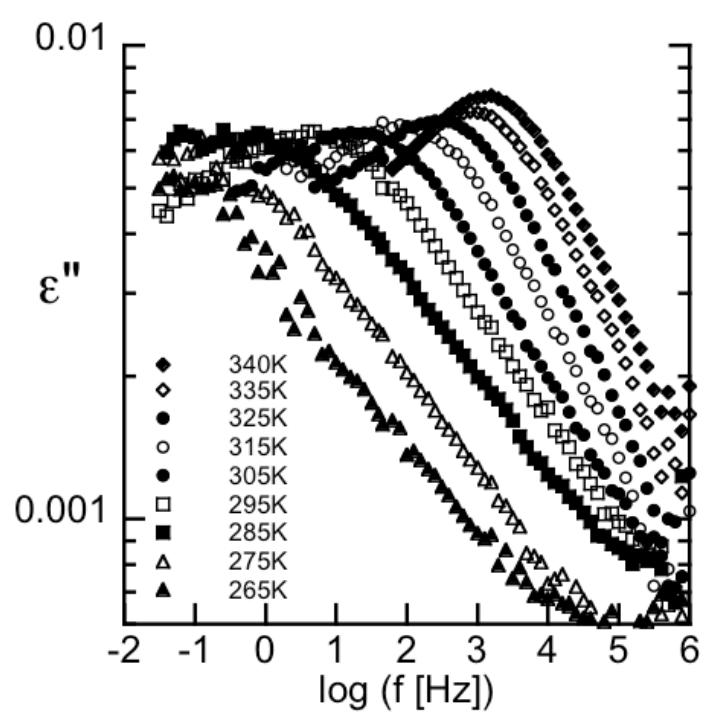

FIG. 7: Imaginary part of the relative dielectric permitivity of 20\%PI27/PtBS23 sample at temperatures between $340 \mathrm{~K}$ and $265 \mathrm{~K}$ where the contribution of the segmental $\alpha$-relaxation has been subtracted.

relaxation for PI concentrations $\geq 35 \%$. For $20 \%$ PI blends, high frequency side HWHM values obtained after subtracting the contribution of the $\alpha$-relaxation deduced from PI765 blends were used. In doing so, on the one hand we confirm that the widths obtained for the $20 \%$ samples after subtraction follow the general trend, and on the other hand, we avoid possible imprint of the PtBS's $\alpha$-relaxation in the results, as this contribution, which locates at the low frequency side of the PI27's NM, may not be completely negligible for the highest PtBS content. 


\section{DISCUSSION}

\section{A. Dynamic asymmetry and chain relaxation in polymer blends}

The Rouse Model is the starting point and standard description for the Brownian dynamics of non-entangled polymer melts ${ }^{29}$. It represents a linear chain as a series of beads and springs subjected to random forces in a medium with constant friction representing the interactions exerted by the surrounding chains. This fully solvable scheme leads to exponentially decaying and orthogonal normal modes for chain dynamics. Although this simple approach obviously fails in describing the melt dynamics of long entangled chains at long times, the Rouse model is also used for describing the fastest part of the response of these long chains, an thereby it is a common ingredient of all available models and theories. The suitability of the Rouse model to describe the chain dynamics of low molecular weight PI $\left(\overline{M_{n}}=2700\right.$ Da just the same used herein) has been carefully checked recently ${ }^{26}$. In contrast, it is clear from previous sections that non-entangled PI chain dynamics in PI/PtBS blends does not follow Rouse's predictions. PI/PtBS blends exhibit high dynamic asymmetry so that the relaxation of PtBS is much slower than that of PI. The presence of such a slow matrix may question a fundamental assumption of the Rouse model, the purely random character of the external forces, bringing memory effects into play.

As shown in the previous section and contrary to the general behavior in non-entangled homopolymers, we observe a non-exponential behavior for the slowest chain relaxation as reflected in the low frequency broadening of the NM-relaxation of PI in PI/PtBS blends. Moreover, the tTS does not hold for either the low or the high frequency sides of the NMrelaxation (see figure 5b for example). The molecular weight of all the samples studied herein is well below the entanglement limit for PI, which is around 4000Da. Therefore, it is 
difficult to imagine an scenario where entanglements, tube reptation, and similar size effects might give account of the measured broadening. We will therefore look for an explanation of the observed features in the high dynamic asymmetry between the different components of the blend.

The existence of two distinct dynamics or effective $T_{g}$ s for the individual components of a miscible blend is nowadays a well established phenomenon. In the Rouse model the viscosity and the monomeric friction coefficient driving terminal dynamics are intimately related to the segmental relaxation. One might expect then, that the progressive freezing of the high- $T_{g}$ component segmental dynamics might have some effect too on the monomeric friction coefficient and the NM-relaxation observed for the low- $T_{g}$ component. In order to quantitatively discuss the effect of the freezing of the slow PtBS component on the NMrelaxation of PI, we need to calculate the expected values for the $\alpha$-relaxation frequencies of PtBS in the blend. This was accomplished from the effective glass transition of PtBS component experimentally measured by calorimetry ${ }^{28}\left(T_{g, e f f}^{P t B S}\right)$, assuming: (i) the same VFT law as for pure PtBS but the $T_{o}$ parameter; and (ii) $T_{g}^{\text {pure }}-T_{g, \text { eff }}^{\text {blend }}=T_{o}^{\text {pure }}-T_{o}^{\text {blend }}$. For high PI27 content samples ( $\gtrsim 65 \%$ ) where the calorimetric $T_{g}$ of the PtBS is not well resolved, the effective $T_{g}^{P t B S}$ was estimated from a Gordon-Taylor extrapolation of the existing values. The suitability of this approach was addressed and discussed in a separate communication ${ }^{28}$.

Figure 9 shows in its upper panel the derivative of the heat flow as a function of inverse $\mathrm{T}$ for the $35 \% \mathrm{PI} 27 / \mathrm{PtBS} 23$ blend $^{28}$. Two well resolved maxima can be observed which correspond to the effective glass transitions of each component in the blend. The lower panel of the figure shows the relaxation map for the same blend. Filled and empty points represent respectively the characteristic $\alpha$ - and NM-relaxation times of PI experimentally measured by DS, while the dashed line represents the characteristic times for the $\alpha$-relaxation of PtBS23 


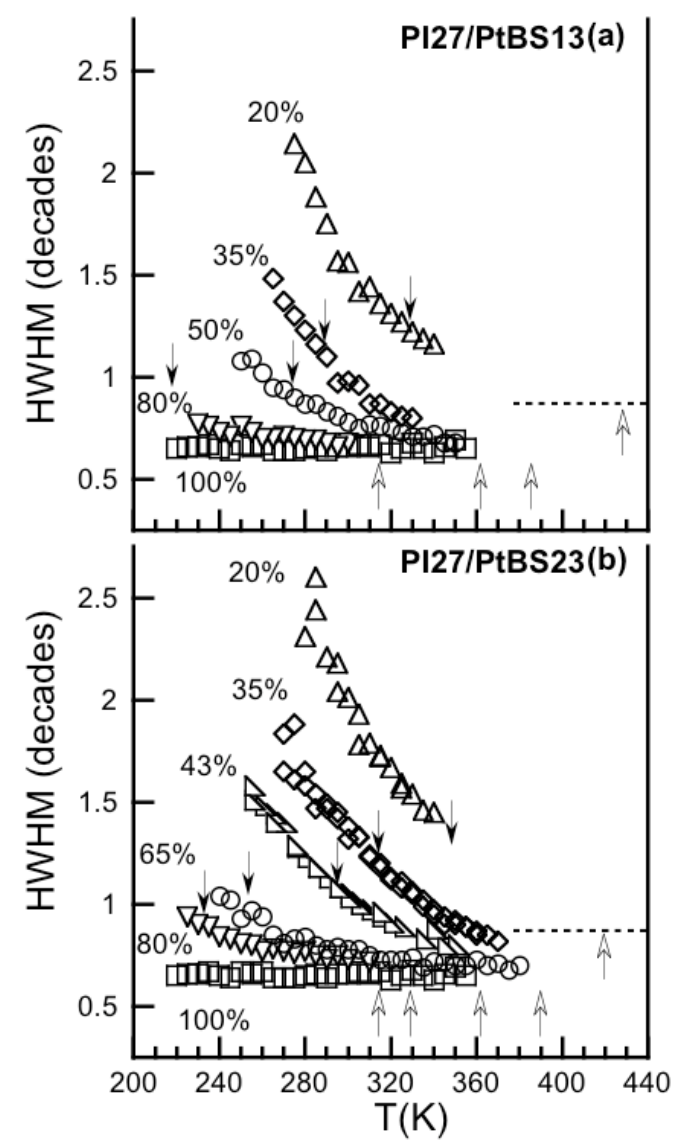

FIG. 8: HWHM at the low frequency side of PI's normal mode in PI27/PtBS13 blends at 35, 50, 80 and $100 \%$ PI content (panel-a) and in PI27/PtBS23 blends at 35, 43, 65, 80 and 100\% PI content (panel-b). The 20\% PI27 blends HWHM data were extracted from the high frequency side after subtracting the contribution from the PI's $\alpha$-relaxation. For these cases, dotted lines represent average HWHM at the high frequency side for pure PI. Arrows mark the temperature where $\tau_{N M-P I} / \tau_{\alpha-P t B S}=100$ (empty arrows) and $\tau_{N M-P I}=\tau_{\alpha-P t B S}$ (filled arrows) for the different set of data. The arrows most at the left correspond to 80PI \% sample and those that follow to the rest of studied concentrations from higher to lower PI \% as we move from left to right.

in the blend obtained by the procedure described above. Note that the extrapolation at 100 


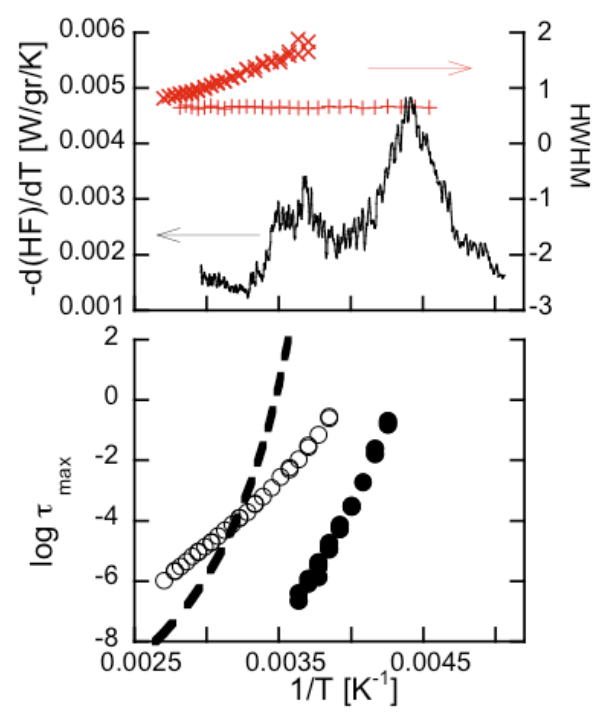

FIG. 9: Panel (a): Solid line represents the derivative of the heat flow with respect to T for a blend $35 \% \mathrm{PI} 27 / \mathrm{PtBS} 23$; crosses and plusses are the HWHM at the low frequency side of the NM for 35\% PI27/PtBS23 and pure PI27 samples, respectively . Panel (b): Characteristic times of the segmental (filled symbols) and NM (empty symbols) relaxations of PI27 in the blend 35\%PI27/PtBS23. Dashed line represents an estimation of PtBS23's $\alpha$-relaxation in the same blend from DSC results in panel (a).

sec. of the characteristic times for the $\alpha$-relaxation of PI measured by DS (solid points) nicely matches the low temperature peak of the calorimetric response. HWHMs of PI's NM in the 35\% PI27/PtBS23 blend are represented in the upper panel of figure 9 by crosses, the HWHMs for pure PI are also included as a reference (plusses). The comparison of different information in figure 9 shows that the departure of the NM width from the value observed at high temperatures (close to that expected for the Rouse model) occurs in the broad range of the PtBS glass transition, and for temperatures where the characteristic time for the PtBS's $\alpha$-relaxation becomes close to or slower than that of PI's NM. This is also the case 
for the rest of the blends studied. In order to show this in a more quantitative way, we have included in figure 8 two sets of arrows for the different blends indicating the temperatures where $\tau_{N M-P I} / \tau_{\alpha-P t B S}=100$ (empty arrows) and $\tau_{N M-P I}=\tau_{\alpha-P t B S}$ (filled arrows). The arrows most at the left correspond to 80PI \% sample and those that follow to the rest of studied concentrations from higher to lower PI $\%$ as we move from left to right. When the characteristic time of PtBS's $\alpha$-relaxation is significantly faster than PI's NM-relaxation there is not significant extra broadening of the NM. Therefore, empty arrows indicating the temperature where PtBS's $\alpha$-relaxation is 100 times faster than the NM, they all locate in the high-T low-HWHM end of the curves. In contrast, when the characteristic times of both processes become alike (filled arrows at $\tau_{N M-P I}=\tau_{\alpha-P t B S}$ ) similar degree of broadening is observed for all the blend compositions.

Let us now look at the previous literature on the subject. The question of whether the terminal relaxation of the components of a blend fulfill the time temperature superposition (tTS) has been the subject of a number of works. Adachi et al. showed tTS for the terminal dynamics of PI in $\mathrm{PVE}^{30}$ and high molecular weight $\mathrm{PBO}\left(\overline{M_{w}}=20500 \mathrm{Da}\right)$ in PVE $\left(\overline{M_{w}}=31000 \mathrm{Da}\right)^{31}$, both measured by dielectric spectroscopy. The experimental temperatures accessed in those works were all below the $T_{g, \text { eff }}$ estimated for the high- $T_{g}$ component by these and others authors for the cited systems ${ }^{15}$. Lodge et al. ${ }^{16}$ reported the breakdown of tTS for PEO's terminal dynamics in diluted blends of PEO/PMMA. The broadenning of PEO's terminal relaxation in the blend followed the same trend as a function of $\mathrm{T}$ and concentration as that observed here for PI. Once again, the correlation between the $T_{g, e f f}$ of the high- $T_{g}$ component and the change in the shape of the terminal relaxation seems to hold. By using the self-concentration value reported for PMMA in PEO/PMMA blends by the same authors $\phi_{\text {self }}^{P M A}=0.27^{32}$, the estimated $T_{g, \text { eff }}$ for PMMA in blends with 50, 20 
and $10 \%$ PEO content are $\sim 300,350$, and $370 \mathrm{~K}$, respectively ${ }^{33}$. Data in reference ${ }^{16}$ were $^{2}$ recorded between 310 and 440K approximately. Thus, PMMA was very slowly moving or frozen for a significant number of temperatures at 10 and 20\% PEO content, and just slightly above $T_{g, e f f}^{P M M A}$ for $50 \% \mathrm{PEO}$. The quantitative change in the PEO relaxation as PMMA matrix freezes is also reflected in the ratio of chain friction coefficient to matrix viscosity shown by the authors in figure 7 of reference ${ }^{16}$. PEO friction tracks the matrix viscosity just at temperatures well above the effective glass transition of the PMMA component in the blend , so that the decoupling between PEO and PMMA becomes more pronounced the lower the temperature and the higher the PMMA content. The behavior in figure 7 of reference ${ }^{16}$ (both $\mathrm{T}$ and concentration) resembles that observed for HWHM in the present work, suggesting that the decoupling of the fast component from the matrix viscosity as the slow component freezes may be a general phenomenology in blends breaking the tTS.

Regarding the PI/PtBS system, Watanabe et al. also studied the terminal relaxation of PI/PtBS blends on samples of higher $M_{w}$ than those studied herein ${ }^{17,34}$. Similar to our results they reported a gradual broadening of the terminal relaxation of PI with increasing PtBS content and decreasing T. However, there is a fundamental difference between both set of results, as high $M_{w} \mathrm{PI} / \mathrm{PtBS}$ blends exhibit a violation of the tTS only in the low frequency tail of the terminal relaxation, whereas the samples studied in this work clearly show a broadening of both, low and high frequency tails of the terminal relaxation. These differences suggest that more than one effect may be behind the broadening of high $M_{w} \mathrm{PI}$ PtBS blends. The comparison of tTS plots for the NM-relaxation of PI in 80\%PI19900/PtBS69500 ${ }^{17}$ (where the numbers after the polymer indicate $\overline{M_{w}}$ in Da units) and PI in 80\%PI19900/PtBS16400 ${ }^{34}$ evidence that the molecular weight plays an important role. While the $80 \% \mathrm{PI}$ blend with PtBS69500 shows clear breakdown of the tTS, that with 
PtBS16400 does not. Furthermore, a PI content as low as 50\% is needed in PtBS16400 containing blends in order to obtain a broadening similar in magnitude to that observed for 80\%PI19900/PtBS69500 blend.

Finally, it is worth mentioning that the broadening of the terminal dynamics of low $M_{w}$ homopolymers has also been reported for various different situations where the motion of the chains was somehow altered by the presence of a more or less "frozen" surrounding medium, like for poly(propylene glycol) in layer-structured Na-vermiculite clay ${ }^{35}$, or poly(propylene oxide) in epoxy reacting blends during network formation ${ }^{36}$.

\section{B. Description in terms of non-exponential modes}

At the light of the previous discussion, it is clear that the observed behavior for the broadening of the NM-relaxation of PI in PI/PtBS should be sought in the high dynamic asymmetry of the system and the gradual freezing of PtBS matrix as temperature decreases. Several possible physical scenarios can be discussed looking for arguments rationalizing the excess broadening of the NM in non-entangled polymer blends. The broadening of the segmental $(\alpha-)$ relaxation function of a polymer component with respect to the pure polymer behavior is often discussed in terms of thermally driven concentration fluctuations (CF) models. These models are based on the idea that the local CF are quasi-stationary near the glass-transition of the blend, i.e., their lifetimes are much longer than the relaxation time of the segmental relaxation. As a consequence, the sample can be considered as divided into subcells of size $V$, each of them with a given composition and a local glass-transition temperature. The relaxation function of a given component inside any of these regions is assumed to be similar to that corresponding to the pure component, and a distribution of relaxation times is assumed as a result of the distribution of local glass-transition tempera- 

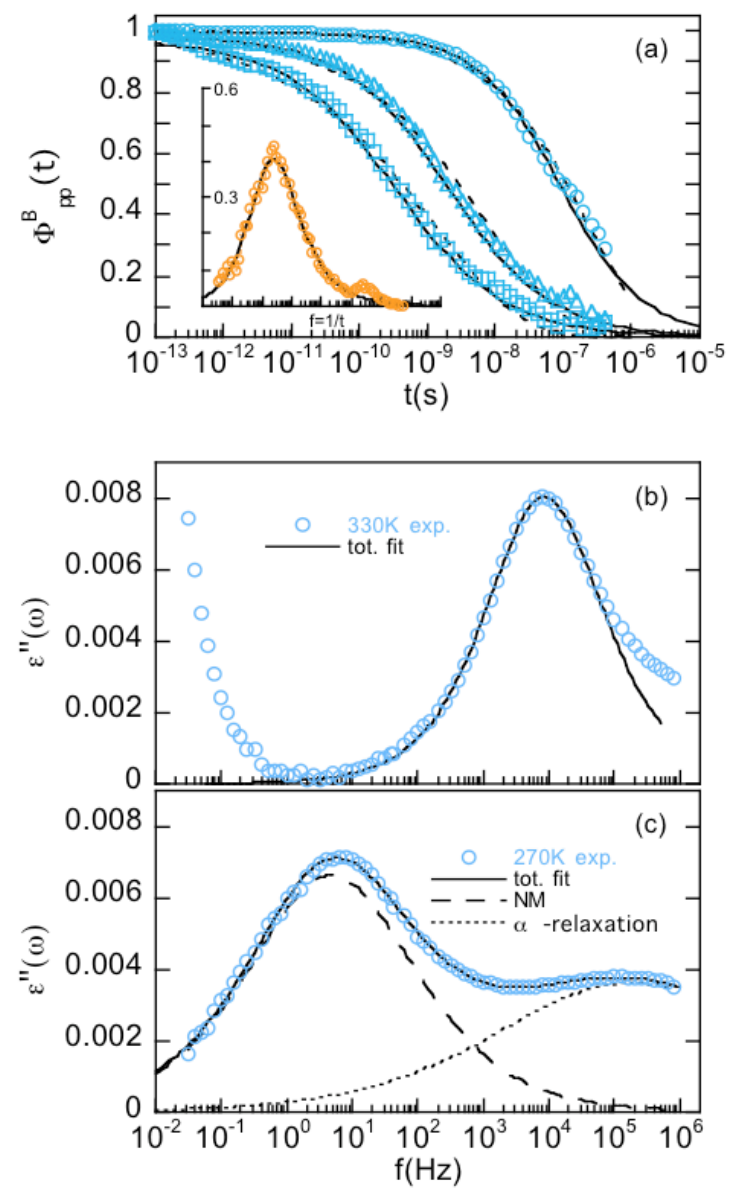

FIG. 10: a) Normalized Rouse correlators $\phi_{p p}(t)$ for the fast component at low temperature ( $\mathrm{T}=0.33$ see reference $\left.{ }^{37}\right)$. Dashed and solid lines represent fits based on KWW and HN functions respectively (see text for details). Inset: bead-spring simulation results in the frequency domain, $\psi(\omega)$ (symbols), for $\mathrm{p}=3$ and $\mathrm{T}=0.44$ ( see reference $^{37}$ ), and its fit to a HN function (solid line). b) and c) Imaginary part of the relative dielectric permitivity of 35\%PI27/PtBS23 blend at 330K and $270 \mathrm{~K}$ respectively (symbols), and its description (solid lines) in terms of a sum of non-exponential modes (see text). Dashed lines in figure c) represent NM and segmental relaxation components.

tures. In principle, CF may also play a role in the broadening of the NM-relaxation function 
of a polymer component with respect to the pure polymer behavior. Several arguments, however, suggest that the effect of $\mathrm{CF}$ in this case might not be as relevant as in the case of the segmental relaxation. On the one hand, it is not clear that the local CF are quasistationary in the timescale of the chain motion, or at least in the timescale of the slower modes. In addition, the characteristic length of the chain motion is much larger than that of the segmental relaxation. As a result, the volume over which concentration fluctuations are probed is larger than for the $\alpha$-relaxation. These two aspects go in the direction of diminishing the degree of heterogeneity experienced by chains both in time and space.

Recent simulation results indicate another scenario for the occurrence of broadened relaxation functions. Simulations of mixtures of short bead-spring polymer chains with high dynamic asymmetry ${ }^{37}$, and fully atomistic simulations of non-entangled PEO/PMMA blends ${ }^{38}$ show just very small deviations from orthogonality between different Rouse modes, but large deviations from the exponential shape of the Rouse modes for the fast component chain dynamics, deviations which increase with decreasing temperature. These phenomena were associated to memory effects induced by the slow nature of the "confining" or slow component, and evidence that the assumption of the time uncorrelation of the stochastic forces breaks down for the fast component, in particular, at the lower temperatures where the dynamic assymetry between the two components in the blend is higher. Together with the non-exponential nature of the relaxation modes, bead-spring simulations also show unusual behavior for the scaling exponent $x$ relating the characteristic times $\tau_{p}$ of the different Rouse modes of a chain $\left(\tau_{p} \sim 1 / p^{x}\right)$. The value of the scaling exponent $x$ gradually varied with temperature from values close to the Rouse scaling at the high temperature limit, i.e. $x \simeq 2.2$, to $x \simeq 3.5$ at the lowest temperature simulated. It is worth noting that these phenomena for short chain bead-spring mixtures can not be essentially attributed to $\mathrm{CF}$ distribution 
effects, as simulations of a single fast chain in a slow matrix (averaged over 100 cells) ${ }^{39}$ lead to analogous phenomenology for the dynamics of the fast component. Therefore, regardless the possible contribution of $\mathrm{CF}$ to the general broadening of chain relaxation functions, according to simulations there exists an underlying and likely dominating mechanism leading to intrinsic non-exponential chain modes.

From a theoretical point of view, the Rouse Model is the starting point and standard description for the Brownian dynamics of non-entangled polymer melts. In order to understand the unsuitability of the Rouse model to describe PI chain dynamics in the blend, one should first check the basic assumptions of the Rouse model. This model (which pre$\operatorname{dicts} \epsilon "(\omega) \sim \omega^{1}$ for the low frequency side of the dielectric NM-relaxation) fully neglects spatial and time correlation of the forces representing the interaction with the surrounding chains. Uncorrelation between forces at different positions leads to orthogonality of the Rouse modes, whereas uncorrelation of the forces at different times leads to exponentially decaying modes. Methods based on generalized Langevin equations (GLE) for the coordinates of the tagged chain encode the interactions with the surroundings in a memory kerne ${ }^{40-42}$ describing the matrix density fluctuations. The Rouse model is obtained as a limit case of GLE when: i) there are no spatial correlations between matrix density fluctuations and ii) the kernel relaxes in a microscopic time scale, i.e., assuming fast decay of matrix density fluctuations. The condition ii) is expected to fail for the fast component in a blend with strong dynamic asymmetry as that investigated here. Interestingly, GLE methods within Renormalized Rouse Models for the memory kernel predict, for slow relaxation of the matrix, the basic phenomenological trends observed for the fast component in the blend. Namely: i) strong stretching (broadening) of the Rouse correlators (response functions) and ii) anomalous scaling of the characteristic times $\tau_{p}$ of the Rouse modes ${ }^{42}$. 
Polymers containing dipole moments along the chain backbone that do not cancel give rise to an "end-to end" net polarization vector. DS gives detailed information of chain dynamics by measuring the dielectric response originated by the reorientation of this net chain dipole. According to the discrete Rouse model for relatively short chains, the correlation function of the end-to-end vector, $\vec{R}$ can be expressed as

$$
\langle\vec{R}(t) \cdot \vec{R}(0)\rangle \propto \sum_{p: o d d}^{N-1} \cot ^{2}\left(\frac{p \pi}{2 N}\right) e^{-t / \tau_{p}}
$$

where $\mathrm{N}$ is the number of beads, and $\tau_{p}$ is the relaxation time associated with the Rouse mode $p$ and is given by

$$
\tau_{p}=\frac{\tau_{N}}{\sin ^{2}(\pi p / 2 N)} .
$$

Equation 2 shows that only the odd Rouse modes contribute to the end-to-end vector correlation function. Moreover, the contribution from the high $p$ numbers is strongly suppressed due to the amplitude factor, which for low $p$ values approximately behaves as $1 / p^{2}$. Therefore, the end-to-end correlation function (and so the dielectric normal mode process) is dominated by the slow (low- $p$ ) odd Rouse modes. The corresponding response function in the frequency domain (that explored by DS) is expressed as

$$
\psi(\omega) \propto \sum_{p: o d d}^{N-1} \cot ^{2}\left(\frac{p \pi}{2 N}\right) \frac{1}{1+i \omega \tau_{p}} .
$$

Equation 2 is only based on the orthogonality of the Rouse modes ${ }^{29}$ and thereby can be generalized for the case where modes do not decay exponentially as long as the orthogonality of the modes is preserved. In this way, the end-to-end vector correlation function can still be expressed in a Rouse-like manner as,

$$
\langle\vec{R}(t) \cdot \vec{R}(0)\rangle \propto \sum_{p: \text { odd }}^{N-1} \cot ^{2}\left(\frac{p \pi}{2 N}\right) e^{-\left(t / \tau_{p}\right)^{\beta}}
$$


where $e^{-\left(t / \tau_{p}\right)^{\beta}}$ is a stretched exponential or Kolraush-William-Watts function (KWW), which is well known for describing nicely many non-exponential processes. Non-Debye processes in the frequency domain, on the other hand, are generally described by a two parameter Havriliak-Negami function $(\mathrm{HN}), 1 /\left(1+(i \omega \tau)^{\alpha}\right)^{\gamma}$. The use in the literature of this phenomenological function to describe dielectric permitivity data is extensive ${ }^{43}$. Parameters $\tau, \alpha$, and $\alpha \gamma$, in the $\mathrm{HN}$ function represent the characteristic time, and the low and high frequency side shape parameters respectively. The function is symmetric when $\gamma=1$, and a Debye expression is recovered when both $\gamma$ and $\alpha$ approach unity. The expression analogue to that in equation 5 in the frequency domain would therefore be,

$$
\psi(\omega) \propto \sum_{p: o d d}^{N-1} \cot ^{2}\left(\frac{p \pi}{2 N}\right) \frac{1}{\left(1+\left(i \omega \tau_{p}\right)^{\alpha}\right)^{\gamma}} .
$$

Coming back to our system, we have seen that the unusual broadening of the NMrelaxation of $\mathrm{PI}$ in $\mathrm{PI} / \mathrm{PtBS}$ blends increases with decreasing temperature and that there is a close relation between the glass transition of the slow PtBS component and the onset of this unusual broadening. The gradual freezing of the slow PtBS component would entail the slow relaxation of the matrix density fluctuations and likely the non-negligibility of time correlations for the external forces on the tagged PI chain. The broadening phenomenology and scenario is analogue to that found in simulations of bead-spring blends in reference ${ }^{37}$, therefore, we will try to describe the dielectric NM-relaxation in terms of a sum of intrinsically non-exponential modes (equation 6), with the same characteristics and scaling laws as those observed in the simulated bead-spring system. In order to have a convenient parametrization of the bead-spring simulation results, we analyzed mode's response functions in the frequency domain by means of HN functions. Consistently, this analysis leads to the same conclusions as those derived from the time domain analysis of data in reference ${ }^{37}$. In practice, the frequency dependent response functions, $\psi(\omega)$, were obtained by applying 
Hamon's approximation ${ }^{44}$ to the normalized Rouse correlators $\phi(t)$ already calculated from the simulation runs,

$$
\left.\left.\psi(\omega) \simeq \frac{\psi(t)}{\omega}\right|_{t=0.63 \omega} \simeq \frac{d \phi(t)}{d \log t}\right|_{t=0.63 \omega}
$$

The inset of figure 10a contains bead-spring simulation results in the frequency domain, $\psi(\omega)$ (symbols), for $p=3$ and low temperature ( $\mathrm{T}=0.4$, see reference ${ }^{37}$ ) and its fit to a HN function (solid line). In the time domain figure 10a shows the $\phi(t)$ data calculated from the results of the simulation runs (symbols), fits of these curves to a KWW (dashed lines), and the curves numerically calculated-back by integrating the HN fits of $\psi(\omega)$ (solid lines). In general a HN description of the $\phi(t)$ data according to equation 7 is better than the KWW approach used $\operatorname{in}^{37}$ (mainly at long times and for very stretched curves), but the scaling laws for the characteristic times obtained from both descriptions agree well. Moreover, when the modes are very wide (low T and high $p$ ) they become quite symmetric and a HN description with $\gamma=$ 1 turns out to be adequate. On the other hand, if a generalized Langevin equation leading to equation 5 existed, there might be a certain relationship between the $\beta$ (or $\alpha, \gamma$ ) shapeparameter of the stretched exponential and the scaling parameter $x$ defining the relation between different $\tau_{p} \mathrm{~s}$ at constant temperature. As we have commented above, simulations results show that $x$ varies gradually with temperature from $x \simeq 2.2$ at high temperatures to $x \simeq 3.5$ at low temperature, while the $\beta$ parameter decreases from values close to 1 at high temperatures to $\sim 0.5$ at the lowest ones. Figure11 shows for the first mode of the bead-spring simulations the relationship between the exponent $x$ and the shape of the mode, which was conveniently parametrized by the full width at half maximum (FWHM) of the curves in the frequency domain.

For the description of PI's NM-relaxation in PI/PtBS blends, model dielectric response functions have been constructed by summing up three HN functions corresponding to modes 


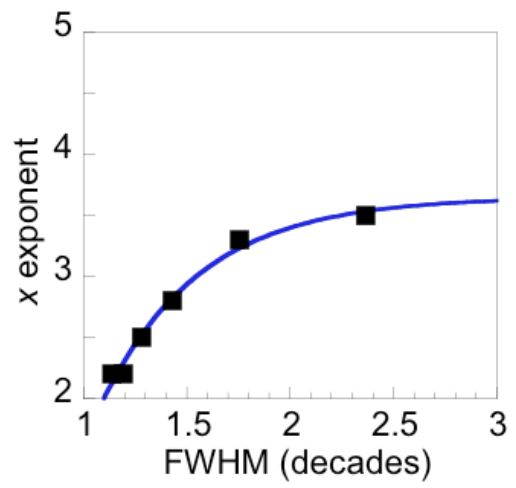

FIG. 11: $x$ parameter as a function of the FWHM of the first mode for the motion of the fast component of the bead-spring blend. Solid line is the result of fitting filled squares. $\mathrm{p}=1,3$, and 5 ,

$$
\epsilon^{\prime \prime}(\omega)=\sum_{p: 1,3,5} \frac{\Delta \epsilon_{1} / p^{2}}{\left(1+\left(i \omega \tau_{1} / p^{x}\right)^{\alpha_{p}}\right)^{\gamma_{p}}} .
$$

In equation 8 we have used the approximation $\cot ^{2}(p \pi / 2 N) \sim p^{-2}$ for $p<<N$. Note that a Rouse's scaling is assumed for the static amplitudes of the modes. This was indeed the observed behavior in simulations ${ }^{37,38}$, where the static properties of the chains are not altered by blending. We have just considered the first three terms of the sum because the contribution of higher modes is strongly suppressed due to the amplitude factor. During the fitting of the experimental NM dielectric data with equation 8 only three parameters remained free $\left(\Delta \epsilon_{1}, \tau_{1}\right.$ and $\left.\alpha_{1}\right)$ and mainly the low frequency side of the curves was used (from low frequencies up to $30 \%$ of the decade above the maxima). The rest of the parameters were expressed as a function of $\Delta \epsilon_{1}, \tau_{1}$ and $\alpha_{1}$ according to the results of the bead-spring simulations. Comparing the shape of different bead-spring simulation modes no significant variation of the ratios $\alpha_{1} / \alpha_{3}, \alpha_{1} / \alpha_{5}, \gamma_{1} / \gamma_{3}$, and $\gamma_{1} / \gamma_{5}$ was observed with temperature. Therefore, these were fixed to an averaged value (over all the available simulation temperatures) $\alpha_{3}=\alpha_{5}=\alpha_{1} / 1.14$ and $\gamma_{3}=\gamma_{5}=\gamma_{1} / 1.14$. Likewise, a relation between $\alpha_{1}$ 
and $\gamma_{1}$ for different Ts was parametrized as $\gamma_{1}=1.95-1.6 \alpha_{1}$ for $\alpha_{1}>0.60$; and $\gamma_{1}=1.0$ for $\alpha_{1}<0.60$, i.e. low temperatures. Finally, the $x$ parameter was expressed in terms of $\alpha$ and $\gamma$ by means of the relationship between $x$ and FWHM in figure 11 and the relationship of FWHM and $\alpha$ and $\gamma$ parameters $(\mathrm{FWHM}=-0.516+1.058 / \alpha+0.039 / \gamma+0.563 /(\alpha \gamma)$, see reference $\left.{ }^{10}\right)$.

Figure 10b shows as an example the dielectric response constructed in this way from a sum of non-exponential (HN) modes (solid line) and the experimental data of a 35\%PI27/PtBS23 sample (symbols) at $\mathrm{T}=330 \mathrm{~K}$. As can be seen, the behavior of the simulated chain is in good agreement with the experimental observation. Note that although the fitting only involved the low frequency side of the curve up to 2-3 points above the maxima, the calculated curve reproduces well the shape of the measured relaxation up to more than one decade above the maxima, up to about half of the maximum intensity. Deviations from the predicted shape at higher frequencies are presumably related with the contribution of the segmental relaxation. Good agreement is also found between the experimental measurements at higher temperatures or higher PI content, and calculated model curves. Experimental curves obtained at low temperatures, as the one shown in figure $10 \mathrm{c}$ at $\mathrm{T}=270 \mathrm{~K}$ for a $35 \% \mathrm{PI} 27 / \mathrm{PtBS} 23$ sample (symbols), exhibit FWHMs in the range $\sim 2.5-3.0$, i.e. beyond the last FWHM point obtained by bead spring simulations at the lowest temperature $(x \sim 3.5$, FWHM $\sim 2.4$ see figure 11). Thus, to fit low temperature curves, as there is no simulation available with such a big broadening, we have assumed the same relations between the shape parameters of the 1st, 3rd and 5th modes, and $x$ parameter values defined by the solid line in figure 11. Note that modes are symmetric at low temperatures, so that $\gamma_{1}=\gamma_{3}=\gamma_{5}=1$. The so obtained curves, where a contribution from segmental relaxation has also been included, describe very well the experimentally observed dielectric normal modes at lower temperatures 
(see for example in figure $10 \mathrm{c}$ ): solid line for total and dashed lines for separated $\alpha$ and NM contributions). From the experimental point of view it is very difficult to distinguish between different broadening scenarios. We have seen that a sum of non-exponential modes in the spirit of Renormalized Rouse Models alone seems to be suitable to describe the experimental dielectric data at all the temperatures analyzed. Nevertheless, and although some arguments (see above) suggest that the relevance of $\mathrm{CF}$ on chain motions might not be as important as for the segmental relaxation, effects attributable to $\mathrm{CF}$ can not be completely ruled out.

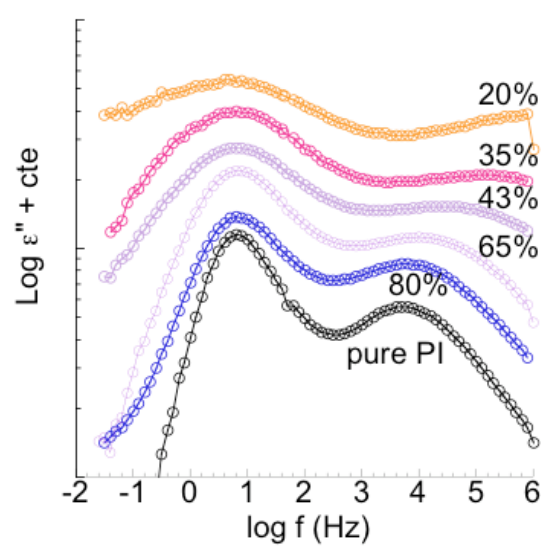

FIG. 12: Imaginary part of the relative dielectric permitivity for PI and PI in PI27/PtBS23 blends at different concentrations and temperatures. Different curves have been shifted in the $y$ axis for clearness.

\section{Relation between segmental and terminal relaxations}

We have shown that both $\alpha$ - and NM-relaxations of PI slow down relative to those observed in the homopolymer when blended with higher $T_{g}$ PtBS. However, as we will show below, the relationship between both phenomena is not trivial. As it can be seen in figure 12, 
the NM- and $\alpha$-relaxation timescales grow more and more away from each other the lower the PI content. The distance between the $\alpha$ - and NM-relaxation for a characteristic frequency of the NM of $1 \mathrm{~Hz}$ ranges from about 3 decades for high PI content to almost 5-6 decades for the $20 \%$ PI blends. Figures 13 and 14 show in a more quantitative way the difference between the characteristic logarithmic frequencies of the $\alpha$ - and NM-relaxations as a function of the NM characteristic frequency of PI in its blends with PtBS13 and PtBS23 respectively. As PI content decreases, the distance between the NM- and $\alpha$-relaxation timescales increases and therefore, the number of points experimentally available for figures 13 and 14 decrease (there are fewer temperatures where we can simultaneously see both maxima in the experimental window). Due to this limitation, in figures 13 and 14 in addition to experimental $f_{\max , \alpha}$ and $f_{\max , N M}$ points (filled symbols), $f_{\max , \alpha}$ values extrapolated from the fitting of experimental values $^{28}$ to a VFT law, vs. experimental $f_{\max , N M}$ points have also been included as empty symbols connected by lines. For PI content higher than 50\%, the slowing down of both $\alpha$ - and NM-relaxations is parallel and the distance remains constant well above $T_{g}$ whereas both relaxations slightly approach each other near $T_{g}$. For these concentrations, the separation between chain and segmental relaxations remains similar to that observed in the pure homopolymer, $\sim 3$ decades at high- $T$ and $\sim 2.5$ decades when approaching $T_{g}$. It could be concluded for these samples then, that both dynamics sense a similar change in the friction coefficient at least at temperatures well above $T_{g}$. For low PI content blends, however, the NM-relaxation is much slower relative to the $\alpha$-relaxation than the behavior observed for the homopolymer. According to the extrapolated VFT values for the $\alpha$-relaxation, the separation between the relaxations for low PI content samples would become more and more pronounced as temperature and PI content decreased even well above $T_{g}$. Regarding the evolution of the separation with temperature (far from $T_{g}$ ), for the higher PI content blends 
the separation remains constant, while the T-dependence dramatically increases for samples below $35 \%$ in PI.

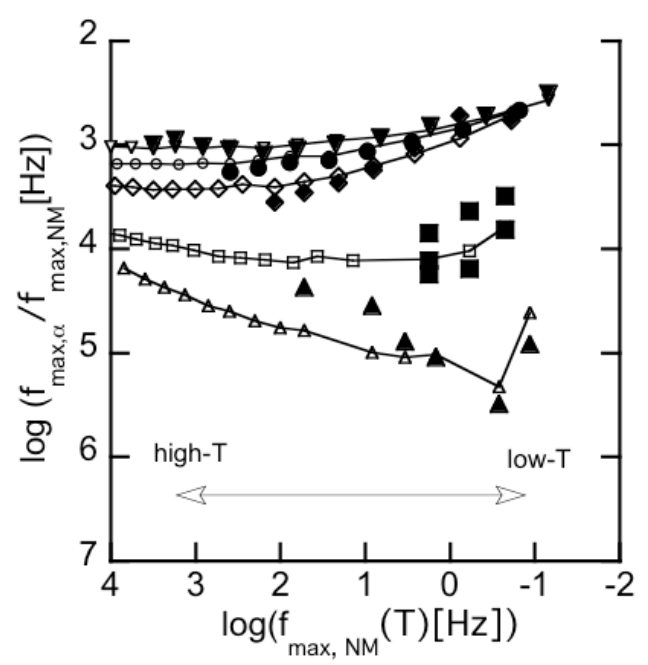

FIG. 13: Distance between the characteristic frequencies of the $\alpha$ - and NM-relaxations of PI component in PtBS13 blends as a function of NM characteristic frequency. Filled symbols: experimental points. Empty symbols: experimental points for the NM-relaxation and VFT fit extrapolations for the $\alpha$-relaxation. Pure PI $(\boldsymbol{\nabla}) ; 80 \% \mathrm{PI}(\bullet) ; 50 \% \mathrm{PI}(\bullet) ; 35 \% \mathrm{PI}(\boldsymbol{\bullet})$; and $20 \% \mathrm{PI}(\boldsymbol{\bullet})$.

For some systems the Lodge-McLeisch model ${ }^{45}$ has been successfully used to predict the terminal dynamics in miscible blends ${ }^{24}$, under the assumption that terminal and segmental dynamics for a given component in a miscible blend are tightly coupled and have the same temperature dependence. However, the systematic test of this assumption shows that this is not always the case. The literature indicates a disparity of behavior among different miscible polymer blends. Good correspondence between the segmental and terminal Tdependence has been found for PI in PVE and PBO in PVE blends ${ }^{18,23,24,30,31}$. For PB in $\mathrm{PB} / \mathrm{PVE}^{15}$, $\mathrm{PI}$ in $\mathrm{PI} / \mathrm{PS}^{19}, \mathrm{PEO}$ in $\mathrm{PEO} / \mathrm{PMMA}^{16,46}$, and $\mathrm{PEO}$ in $\mathrm{PEO} / \mathrm{PVAc}^{25}$ blends, however, significant difference was found between the T-dependence of terminal relaxation 
times an that of segmental relaxation times. The reasons for the decoupling of terminal and segmental data remain unclear. It has been speculated that the difference between these two groups of blends could rely on the different techniques used to characterize them, as those measured by DS to that date showed good correlation between segmental and terminal dynamics while those measured by NMR did not. The DS data presented in this work rule out this possibility. Other approaches have suggested that the segmental motions of the two components of a blend might weakly couple to each other if the two homopolymers have large structure disparity, whereas strong coupling should always exist in the terminal dynamics of all miscible polymer blends ${ }^{15}$. Our high- $T_{g}$ component has a rather large side group and the self-concentration in the blend is quite large $\left(\phi_{\text {self }}^{P I} \sim 0.6^{28,47}\right)$, so that the "weak coupling" view would in principle be compatible with our data in PI/PtBS. However, other systems can be mentioned where the reported $\phi_{\text {self }}$ for the fast component is low and different T-dependence for the segmental and terminal relaxations have been observed, as for PEO in PVAc ${ }^{25}$ or PI in miscible blends with $\mathrm{PS}^{19}$.

We note that the onset of an unusual broadening of the terminal relaxation of PI in PtBS blends, and the decoupling of the terminal and segmental relaxation times of PI both show similar trends with temperature and composition. Moreover, a significant number of systems which do not show correspondence between fast component segmental and terminal dynamics have been reported to break down the tTS and the other way round ${ }^{16,18,23-25,30,31}$. It seems quite reasonable then to consider that these two phenomena might be related and brought about by the slowing down of the high- $T_{g}$ component segmental dynamics. In this line, Zhao et al. in a recent work ${ }^{25}$ reported that the decoupling of the terminal and segmental relaxation becomes more pronounced as the dynamic asymmetry increases.

As we have already commented, associated to memory effects induced by the slow nature 


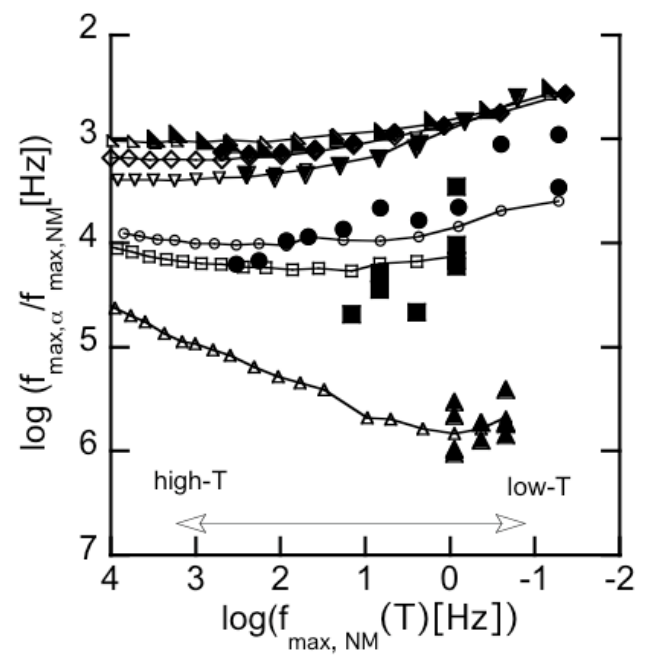

FIG. 14: Distance between the characteristic frequencies of the $\alpha$ - and NM-relaxations of PI component in PtBS23 blends as a function of NM characteristic frequency. Filled symbols: experimental points. Empty symbols: experimental points for the NM-relaxation and VFT fit extrapolations for the $\alpha$-relaxation. Pure PI $(\bullet) ; 80 \% \mathrm{PI}(\bullet) ; 65 \% \mathrm{PI}(\boldsymbol{\nabla}) ; 43 \% \mathrm{PI}(\bullet) ; 35 \% \mathrm{PI}(\boldsymbol{\square}) ;$ and $20 \% \mathrm{PI}(\boldsymbol{\Delta})$. of the "confining" slow component, simulations of mixtures of short bead-spring polymer chains with high dynamic asymmetry show : i) large deviations from the exponentiality of the fast component terminal relaxation; and ii) breakdown of the Rouse scaling $(x \simeq 2)$ as temperature decreases. The relation between the normal mode and segmental relaxation time (the latter taken as the time of the fastest mode, i.e. that with the shortest wavelength) for simulated bead spring blends and its comparison with the experimental results found for $\mathrm{PI}$ in PtBS is also stimulating. As can be seen in figure 15, simulated data show the same trend observed in the experiments: the distance between the two processes remains constant for the homopolymer (filled symbols) but decreases with temperature for the low- $T_{g}$ chains in the blend (empty symbols). More interestingly, this behavior can be well correlated with the deviation of the scaling exponent $x$ from the predicted Rouse behavior. Simulations 
have the advantage that provide a relatively easy access to the information of individual modes and the $x$ scaling parameter, which is by no means a trivial issue when analyzing experimental data. As seen in figure 15, for the homopolymer in the whole temperature range simulated and for the low- $T_{g}$ component at high temperatures, the distance between segmental and NM processes remains more or less constant and the scaling exponent is $x \simeq 2.2$, very close to the Rouse value $x=2$. This is not surprising as at high temperatures, where dynamic asymmetry vanishes, it is expected that the assumptions of the Rouse model, that is the random character of the forces, are well fulfilled for the blend too, so that chain dynamics agrees with Rouses predictions. At lower temperatures, however, we observe a parallel increase of both the separation of segmental and NM-relaxation times and the scaling exponent $x$. The increase of the scaling exponent $x$ is an indicator of the gradual change of dynamic behavior from Rouse like to another regime where time uncorrelation of the forces is not negligible (leading to non-exponentiality of the Rouse modes). Therefore, these results point once again to a close relation between all these phenomena: the unsuitability of the Rouse model, the gradual broadening of the NM-relaxation for the fast component of blends with high dynamic asymmetry, the wide and T-dependent separation between segmental and NM-relaxation, the anomalous dynamic scaling of Rouse modes $(x(T) \geq 2)$ despite the regular scaling for static properties, and the freezing of the high- $T_{g}$ component.

\section{CONCLUSIONS}

We have studied the PI component dynamics in PtBS blends of low molecular weight over the entire composition range. Dielectric response of PI becomes slower as the high- $T_{g}$ component PtBS increases. Moreover, there is a gradual and quite symmetric broadening of both low and high frequency tails of the NM-relaxation as PtBS\% increases and for a given 


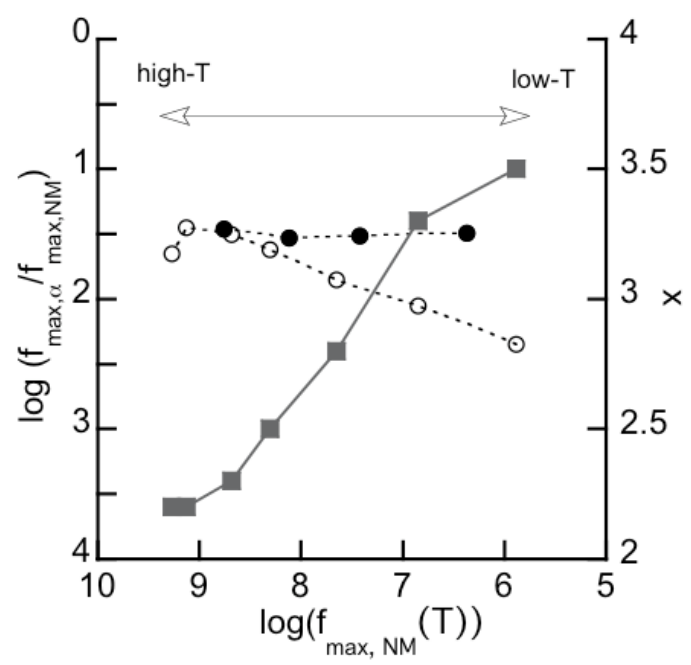

FIG. 15: Left axis: distance between the characteristic frequencies of the $\alpha$ - and NM-relaxations for the fast component of a bead-spring blend (empty circles) and the same magnitude for the corresponding homopolymer (filled circles). Right axis: scaling exponent $x$.

composition as temperature decreases. Previous reports of tTS breakdown for the same system but higher molecular weight had been reported to occur only at the high frequency tail of the NM-relaxation. The magnitude of the here reported broadening depends on: i) the concentration; ii) the temperature; iii) the dynamic asymmetry, i.e. the difference between the components' pure $T_{g}$. In addition, the onset of the mentioned broadening correlates well with the slowing down of the segmental relaxation of the high- $T_{g}$ PtBS component. The fact that the molecular weight of the components is well below the entanglement limit together with the correlation mentioned above, exclude more habitual interpretations based on topological size effects and points to other factors like dynamic asymmetry in blends and its effects.

A sum of non-exponential modes following the scaling laws observed in bead-spring simulations of polymer mixtures with high dynamic asymmetry seems to be adequate to describe 
the experimental dielectric data at all the temperatures analyzed. On the other hand, the segmental and chain dynamics of the PI component in the blend show different Tdependence even well above $T_{g}$. As a result, the separation between the maxima of both relaxations ranges from 3 decades in pure PI and PI $\geq 50 \%$ content blends, to 5-6 decades for $20 \%$ PI blends. This behavior is also qualitatively reproduced in simulations of bead-spring blends, which allow to correlate this feature with a parallel increase of the scaling exponent $x$. All these results strongly point to a close relation between the unsuitability of the time uncorrelation assumption for the forces in the Rouse model, the gradual broadening of the NM-relaxation for the fast component, the large and T-dependent separation between segmental and NM-relaxation, the anomalous dynamic scaling of Rouse modes $(x(T) \geq 2)$ despite the regular scaling for static properties, and the freezing of the high- $T_{g}$ component in blends with high dynamic asymmetry.

\section{Acknowledgments}

We acknowledge the support of the following research projects, contracts, and grants: DYNACOP PEOPLE-2007-1-1-ITN ref: 214627 by the European Union; MAT-2007-63681 by the Spanish Ministerio de Educación y Ciencia; and IT-436-07 by the Basque Government.

1 ed: Koza, M.; Frick, B.; Zorn, R. Proceeding of the 3rd International Workshop on Dynamics in Confinement. Eur. Phys. J. - Special topics., 2007, 141.

2 Colmenero, J; Arbe, A. Soft Matter, 2007, 3, 1-13.

3 Gill, L.; Damron, J.; Wachowicz, M.; White, J. L. Macromolecules 2010, 43, 3903.

4 Sy J. W.; Mijovic J. Macromolecules 2000, 33, 933. 
${ }^{5}$ Koizumi S. J. Polym. Sci., Part B: Polym, Phys. 2003, 42, 3148.

${ }^{6}$ Wolak, J.; Jia, X.; Gracz, H.; Stejskal, E. O.; White, J. L.; Wachowicz, M.; Jurga, S. Macromolecules 2003, 36, 4844.

7 Genix A. -C.; Arbe A.; Alvarez F.; Colmenero J.; Willner L.; Richter D.. Phys. Rev. E 2005, 72,031808 .

8 Lorthioir, C.; Alegría, A.; Colmenero, J. Phys. Rev. E 2003, 68, 031805.

${ }^{9}$ Roland C. M.; McGrath K. J.; Casalini R. Macromolecules 2006, 39, 3581.

10 Schwartz G. A.; Colmenero J.; Alegría A. Macromolecules 2007, 40, 3246.

11 Tyagi M.; Arbe A.; Alegría A.; Colmenero J.; Frick B. Macromolecules 2007, 40, 4568.

12 Swenson J.; Bergman R.; Longville S. J. Chem. Phys. 2001, 115, 11299.

13 Cerveny S.; Barroso-Bujans F.; Alegría A.; Colmenero J. J. Phys. Chem. C 2010, 114, 2604.

14 Schonhals A.; Göering H.; Schick Ch.; Frick B.; Zorn R. J. of Non-Cryst. Solids 2005, 351 2668.

15 He Y.; Lutz T. R.; Ediger M. D. Macromolecules 2004, 37, 9889.

16 Zeroni I.; Ozair S.; Lodge T. P. Macromolecules 2008, 41, 5033.

17 Watanabe, H.; Matsumiya, Y.; Takada, J.; Sasaki, H.; Matsushima, Y.; Kuriyama, A.; Inoue, T.; Anh, K. H.; Yu, W.; Krishnamoorti, R., 2007, 40, 5389-5399.

18 Haley J. C.; Lodge T. P.; He Y.; Ediger M. D.; von Meerwall E. D.; Mijovic J. Macromolecules 2003, 36, 6142 .

19 Lutz T. R.; He Y.; Ediger M. D.; Pitsikalis M.; Hadjichristidis N. Macromolecules 2005, 38, 6216.

20 Ding Y. F.; Sokolov A. P. Macromolecules 2006, 39, 3322.

${ }^{21}$ Plazek D. J. Polym. Sci., Polym. Phys. Ed. 1982, 20, 729. 
22 Rouse E. P. J. Chem. Phys. 1953, 21, 1272.

23 Pathak J. A.; Kumar S. K.; Colby R. H. Macromolecules 2004, 37, 6994.

24 Haley J. C.; Lodge T. P. Journal of Rheology 2004, 48, 463.

25 Zhao J.; Zhang L.; Ediger M. D. Macromolecules 2008, 41, 8030.

26 Clement R.; Alegría A.; Tordjeman P.; Colmenero J. Macromolecules 2009, 42, 8492.

27 K. Yurekli, R. Krishnamoorti. J. Polym. Sci., Part B: Polym. Phys. 42, 3204 (2004).

28 Arrese-Igor S.; Alegría A.; Colmenero J. Macromolecules 2010, 43, 6406.

29 Doi M.; Edwards S. F. The Theory of Polymer Dynamics. Oxford Science Publications. ISBN0-19-852033-6.

30 Hirose Y.; Urakawa O.; Adachi K. Macromolecules 2003, 36, 3699.

31 Hirose Y.; Adachi K. Macromolecules 2006, 39, 1779.

32 Gaikwad A. N.; Wood E. R.; Ngai T.; Lodge T. P. Macromolecules 2008, 41, 2502.

$33 T_{g, e f f}$ was estimated by means of the Lodge and McLeish model. Lodge P. L.; McLeish T. C. B. Macromolecules 2000, 33, 5278 .

34 Chen Q.; Matsumiya Y.; Masubuchi Y.; Watanabe H.; Inoue T. Macromolecules 2008, 41, 8694.

35 Schwartz G. A.; Bergman R.; Swenson J.; Journal of Chemical Physics 2004, 120, 5736.

36 Miijovic J.; Sy J. W. Journal of Non-Crystalline Solids 2002, 307-31, 679.

37 Moreno A. J.; Colmenero J. Phys. Rev. Lett. 2008, 100, 126001.

38 Brodeck M.; Alvarez F.; Moreno A. J.; Colmenero J.; Richter D. Macromolecules 2010, 43, 3036-3051.

39 Moreno A. J. et al. unpublished.

40 K. Schweizer, M. Fuchs, G. Szamel, M. Guenza, H. Tang. Macromol. Theory and Simul. 1997, 
$6,1037-1117$.

41 K. Schweizer. J. Chem. Phys. 1989, 91,5802.

42 R. Kimmich, N. Fatkullin. Adv. Polym. Sci. 2004, 170,1-113.

43 Kremer F.; Schønhals A. Broadband Dielectric Spectroscopy QC454.B74 B76 2002. ISBN 3540434070. Springer- Verlag, Berlin Heidelberg.

44 Hamon B. V. Proc. Inst. Elec. Engrs. London 1952, 99, 115.

45 Lodge T. P.; McLeish T. C. B. Macromolecules 2000, 33, 5278.

46 Lutz T. R.; He Y. Y.; Ediger M. D.; Cao H. H.; Lin G. X.; Jones A. A. Macromolecules 2003, 36, 1724.

47 Zhao J.; Ediger M. D.; Sun Y.; Yu L. Macromolecules 2009, 42, 6777. 


\section{FIGURE CAPTIONS}

Figure 1: Imaginary part of the relative dielectric permitivity of pure PI27 and its blends with PtBS13 at different weight percentages at $280 \mathrm{~K}$.

Figure 2:Imaginary part of the relative dielectric permitivity of 20\% PI765 blend with PtBS23 at different temperatures.

Figure 3: Logarithm of the frequency at the maxima of the normal mode relaxation of pure PI27 and its blends with PtBS13 (a) and PtBS23 (b) at different PI weight percentages.

Figure 4: Parameters for the VFT fitting of the NM relaxation of PI in its blends with PtBS13 (empty symbols) and PtBS23 (filled symbols) at various concentrations.

Figure 5:Imaginary part of the relative dielectric permitivity of: (a) 35\%PI27/PtBS23 blend at temperatures between 360K and 280K; and (b) 43\%PI27/PtBS23 blend normalised to maxima position and intensity.

Figure 6: Imaginary part of the relative dielectric permitivity for 20\%PI/PtBS23 blends with two different PIs: low molecular weight PI27 (empty circles) and high molecular weight PI765 (empty squares) at 270 and 280K. Filled circles represent the dielectric response of the low molecular weight 20\%PI/PtBS23 blend after subtracting the $\alpha$-relaxation from high molecular weight sample.

Figure 7: Imaginary part of the relative dielectric permitivity of 20\%PI27/PtBS23 sample at temperatures between $340 \mathrm{~K}$ and $265 \mathrm{~K}$ where the contribution of the segmental $\alpha$ relaxation has been subtracted.

Figure 8: HWHM at the low frequency side of PI's normal mode in PI27/PtBS13 blends at 35, 50, 80 and 100\% PI content (panel-a) and in PI27/PtBS23 blends at 35, 43, 65, 80 and $100 \%$ PI content (panel-b). The 20\% PI27 blends HWHM data were extracted from the high frequency side after subtracting the contribution from the PI's $\alpha$-relaxation. For these 
cases, dotted lines represent average HWHM at the high frequency side for pure PI. Arrows mark the temperature where $\tau_{N M-P I} / \tau_{\alpha-P t B S}=100$ (empty arrows) and $\tau_{N M-P I}=\tau_{\alpha-P t B S}$ (filled arrows) for the different set of data. The arrows most at the left correspond to 80PI $\%$ sample and those that follow to the rest of studied concentrations from higher to lower PI $\%$ as we move from left to right. 
Figure 9: Panel (a): Solid line represents the derivative of the heat flow with respect to T for a blend 35\%PI27/PtBS23; crosses and plusses are the HWHM at the low frequency side of the NM for 35\%PI27/PtBS23 and pure PI27 samples, respectively. Panel (b): Characteristic times of the segmental (filled symbols) and NM (empty symbols) relaxations of PI27 in the blend 35\%PI27/PtBS23. Dashed line represents an estimation of PtBS23's $\alpha$-relaxation in the same blend from DSC results in panel (a).

Figure 10: a) Normalized Rouse correlators $\phi_{p p}(t)$ for the fast component at low temperature $\left(T=0.33\right.$ see reference $\left.{ }^{37}\right)$. Dashed and solid lines represent fits based on KWW and HN functions respectively (see text for details). Inset: bead-spring simulation results in the frequency domain, $\psi(\omega)$ (symbols), for $\mathrm{p}=3$ and $\mathrm{T}=0.44$ ( see reference $^{37}$ ), and its fit to a HN function (solid line). b) and c) Imaginary part of the relative dielectric permitivity of $35 \% \mathrm{PI} 27 / \mathrm{PtBS} 23$ blend at $330 \mathrm{~K}$ and $270 \mathrm{~K}$ respectively (symbols), and its description (solid lines) in terms of a sum of non-exponential modes (see text). Dashed lines in figure c) represent NM and segmental relaxation components.

Figure 11: $x$ parameter as a function of the FWHM of the first mode for the motion of the fast component of the bead-spring blend. Solid line is the result of fitting filled squares.

Figure 12: Imaginary part of the relative dielectric permitivity for PI and PI in PI27/PtBS2300 blends at different concentrations and temperatures. Different curves have been shifted in the $y$ axis for clearness.

Figure 13:Distance between the characteristic frequencies of the $\alpha$ - and NM-relaxations of PI component in PtBS13 blends as a function of NM characteristic frequency. Filled symbols: experimental points. Empty symbols: experimental points for the NM-relaxation and VFT fit extrapolations for the $\alpha$-relaxation. Pure PI ( $) ; 80 \% \mathrm{PI}(\bullet) ; 50 \% \mathrm{PI}(\bullet) ; 35 \% \mathrm{PI}$ $(\mathbf{\square})$; and $20 \%$ PI $(\mathbf{\Delta})$. 
Figure 14:Distance between the characteristic frequencies of the $\alpha$ - and NM-relaxations of PI component in PtBS23 blends as a function of NM characteristic frequency. Filled symbols: experimental points. Empty symbols: experimental points for the NM-relaxation and VFT fit extrapolations for the $\alpha$-relaxation. Pure PI $(\bullet) ; 80 \% \mathrm{PI}(\boldsymbol{\vee}) ; 65 \% \mathrm{PI}(\mathbf{\nabla})$; 43\%PI (•); 35\%PI (ם); and 20\%PI (ム).

Figure 15: Left axis: distance between the characteristic frequencies of the $\alpha$ - and NMrelaxations for the fast component of a bead-spring blend (empty circles) and the same magnitude for the corresponding homopolymer (filled circles). Right axis: scaling exponent $x$ 


\section{FOR TABLE OF CONTENTS USE ONLY}

"Effect of blending on the chain dynamics of the "Low-Tg" component in non-entangled and dynamically assymetric polymer blends"

Silvia Arrese-Igor, Ángel Alegría, Ángel José Moreno, and Juan Colmenero
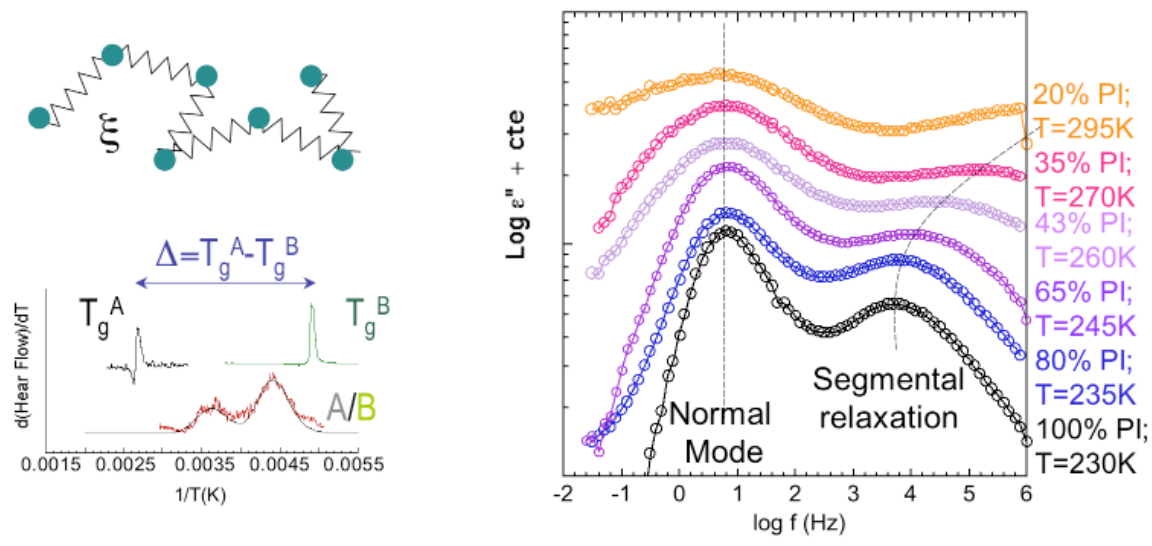